\title{
Recommendations for Gynecological Endoscopic Surgery during COVID-19 Pandemic
}

\author{
Amrutha Kakollu',® Anupama Hari² \\ 1Department of Obstetrics and Gynecology, KIMS Hospital, \\ Secunderabad, Telangana, India \\ ${ }^{2}$ Department of Obstetrics and Gynecology, Malla Reddy College, \\ Telangana, India \\ Ind J Car Dis Wom:2020;5:239-242
}

\begin{abstract}
Address for correspondence Amrutha Kakollu, MS, Fellow in Endogynecology, FMAS, Fellow, Obstetrics and Gynecology, KIMS Hospital, Secunderabad, India (e-mail: amrutha9jaideep@gmail.com).
\end{abstract}

\begin{abstract}
Keywords

- COVID-19

- gynecological

- hysteroscopy

- laparoscopy

The presence of coronavirus disease 2019 (COVID-19) continues to overwhelm health-care systems with numerous concerns around the safety of patients as well as health-care professionals. It is essential to allow for judicious use of resources and emphasize allotting maximum staff and resources to care for the affected. To this end, most centers are choosing to defer elective procedures and perform only emergency surgeries. The safety of all theater staff is of utmost importance, and the risks of severe acute respiratory syndrome coronavirus 2 (SARS-CoV-2) viral transmission should be curbed when operating. In particular, there are concerns relating to the transmission of COVID-19 during gynecological laparoscopic surgery, arising from the possible generation of contaminated aerosols from gas leakage and the creation of smoke from the use of electrosurgical devices. The aim of this paper is to review the evidence available as of today for recommendations to follow while performing gynecological procedures.
\end{abstract}

\section{Introduction}

It's that desperate time for the whole world that calls for desperate measures. Endoscopic surgery, which is extremely popular in terms of being highly beneficial to the patient today, is under the radar with regard to safety of health-care professionals. In this article, we have attempted to answer a few quintessential questions of today: Should we choose traditional open surgery over laparoscopic surgery for minimizing the risk of exposure to health-care professionals? How can we reduce the risk of exposure while performing minimal access surgery?

To justify, safety of the patients and the health-care professionals are equally important and need to be balanced to avoid an unforeseen disintegration of the entire health-care system that could in turn lead to a severe loss of health and life.

\section{General Recommendations}

\section{Preoperative Screening/Testing}

The recommendation by American Association of Gynecologic Laparoscopists (AAGL) is that all patients need to undergo clinical screening before surgery and testing should be done in symptomatic and at-risk patients for coronavirus disease 2019 (COVID-19). ${ }^{1}$ They also recommend the consideration of a patient with unknown COVID-19 status as positive unless proved otherwise, and advocate the measures of universal precautions and provision of adequate protective equipment to the health-care professional.

Chest imaging by computed tomography (CT chest) and reverse transcription polymerase chain reaction (RT-PCR) have been recommended to be ideally individualized, based on the symptoms and risk factors associated with the patient. ${ }^{2}$ Being cognizant of the Indian scenario, both CT chest screening and RT-PCR can be used as complementary investigations to increase the sensitivity and specificity of diagnosing infection, especially if planning for an endoscopic surgery that needs general anesthesia (GA).

There is debatable data on the choice of diagnostic test. However, the RT-PCR has been described as the standard diagnostic test in view of its higher sensitivity. Majority of studies reported a sensitivity of RT-PCR between 70 and $88 \%^{3-6}$ and had included more symptomatic patients with moderate and severe diseases. The sensitivity of RT-PCR is influenced greatly by the technique and site of taking swab, technological aspects of testing. The false negative rate of RT-PCR has been reported up to $15 \%$. This implies that a negative test cannot exclude the disease in all patients. ${ }^{7}$
DOI https://doi.org/ 10.1055/s-0040-1716818.
(C)2020 Women in Cardiology and Related Sciences
License terms

(요 (1) $\Theta \circledast$ 
The role of CT chest cannot be taken lightly. It can be resourceful especially in an emergency and in centers where practical difficulties are encountered in performing a nasopharyngeal swab test for RT-PCR. A systemic review and meta-analysis conducted by Buyun et al on the role of chest CT in screening patients reported a sensitivity of 61 to $98 \%$ and a low specificity of 25 to $33 \%{ }^{8}$

Postoperative outcome may be guarded in asymptomatic COVID-19 patients undergoing surgery or patients who may be in the incubation period of the disease as surgery may alter the course of disease depending on patient's immunity and age. ${ }^{2}$

\section{Anesthesia}

Laparoscopic procedures require GA which is an aerosol-generating procedure (AGP). The risk of transmission to the health-care worker is high during AGP. ${ }^{9}$

The following precautions can be followed during induction of GA.

1. Avoid GA in patients who are COVID-19 positive. Alternatively, these patients can be postponed where applicable and offered laparotomy or vaginal surgery under regional anesthesia if they need surgery.

2. During induction, limit people in operation theater (OT) to the anesthetist and required technicians only during intubation with usage of personal protective equipment (PPE) III.

3. Using a box over the patient's head can reduce the spread of aerosol.

4. Surgical team should enter the theater 20 minutes after induction of anesthesia. ${ }^{9}$

5. Limit movement of people in and out of the OT until completion of surgery and extubation. The operating members can leave the OT prior to the extubation. ${ }^{1}$

\section{Elective Surgery}

Medical treatment where appropriate can be offered to minimize the burden on health care. If surgery is unavoidable then it's essential to follow preoperative clinical and laboratory screening prior to planning surgery. If the patient is positive or a suspect on clinical screen, it is ideal to defer the surgery for 14 days and plan retesting. The patient should be advised home quarantine and follow-up.

\section{Emergency Surgery}

Patients with unknown COVID-19 status: In such patients, preoperative screening and testing help to allocate designated postoperative care and minimize further exposure to other inpatients and educate their family as well.

Confirmed or suspected COVID-19 cases: All standard precautions are to be followed by the entire team. Ideally, emergency laparoscopic procedures in these patients should be performed by an experienced surgeon to ensure safe techniques and minimize the duration of surgery. ${ }^{10}$

\section{Mode of Surgery}

When in doubt and in situations where all the necessary precautions for a laparoscopy cannot be followed, it is imperative to opt for laparotomy or vaginal route for the safety of the patient and the health-care professional.

\section{Safety of the Operating Team}

Anegative pressure environment has been ideally recommended to prevent dissemination of the airborne virus particles beyond the theater. However, implementing this is not possible in all COVID-19 hospitals. Making sure that air handling units in the theater are in working condition with periodic air change and ventilation when operating can be done to reduce the viral particle load in the theater environment. ${ }^{9}$ The air conditioner should be started after induction of anesthesia and stopped 20 minutes before extubation. ${ }^{11}$

Emphasis should be placed on proper surgical techniques that aim to minimize the duration of surgery, generation of surgical smoke and contact with body fluids. ${ }^{10}$ Restricting the number of people inside the theater during procedure is recommended. All personnel should be well versed with the donning and doffing techniques of PPE.

\section{Aerosol and Surgical Smoke}

The highest risk of dissemination has been reported due to the because of aerosol generation at intubation and extubation during GA, as the virus is usually present in the respiratory tract. ${ }^{10}$

Respiratory mode of spread of COVID-19 from airborne virus particles is well documented. This raises the concerns of spread of infection from inhalation of surgical smoke during the use of electrosurgery and $\mathrm{CO}_{2}$ used for the creation and maintenance of pneumoperitoneum. ${ }^{12-14}$ Surgical smoke has been demonstrated to have viral deoxyribonucleic acid, but the inhalation of smoke resulting in disease has not been adequately evaluated. There are reports of identifying human papilloma virus, human immunodeficiency virus, and hepatitis B virus in surgical smoke, but their infectivity is unknown and hence universal precautions are recommended during surgery. ${ }^{\text {? }}$

There is no robust data to demonstrate the presence of COVID-19 in surgical smoke or evacuated $\mathrm{CO}_{2}$ laparoscopic surgery. However, the above data needs to be extrapolated and the leakage of smoke and $\mathrm{CO}_{2}$ into the atmosphere should be addressed by targeted efforts.

However, the enormity of the viral load in surgical smoke during gynecological endoscopic surgery has been considered less than that seen during surgeries on the respiratory tract and gastrointestinal surgeries. RCOG mentions that the proper use of PPE does not increase this risk during gynecological laparoscopic surgeries. ${ }^{10}$ The virus, however, was not identified in genital tract or urine of women from COVID-19, suggesting theoretically lesser risk of transmission via the vaginal route..$^{15,16}$

\section{Minimizing the Aerosol Leakage into Theater Environment}

- Use a proper method of smoke evacuation.

- Ensure that all the trocars being used are leak proof and no cracked valves.

- Use closed entry with Veress rather than open method as the incision of fascia can be smaller reducing gas leakage. 
- Use smaller skin incisions to enable snug fitting of the ports that can reduce slippage of port during manipulation and leak of gas from around the port.

- If excessive slippage of port during procedure, one can consider fixing the port to anterior abdominal wall with a suture.

- Close the cannula valve before connecting and before removing the insufflation tube.

- Have a designated person on the team and a designated port preferably for evacuation during the surgery.

- Deflate the abdomen completely prior to specimen removal either from the abdomen or by colpotomy and before port removal.

- During hysterectomy, to avoid gas leakage from the vault, a vaginal closure can be done or a snugly fitting vaginal tube should be used.

- Do not vent gas from the cannula valve randomly during surgery.

- Do not deflate the abdomen all of a sudden during or after the procedure.

Smoke evacuation and filtration:

There are a number of commercially available smoke evacuators that are recommended and can used with good efficacy. However, they are expensive and add to the cost of surgery along with the preoperative screening, usage of PPE. The $\mathrm{CO}_{2}$ used in pneumoperitoneum and surgical plume generated during surgery may contain viral particles facilitate the dispersion of virus from the abdomen of the patient to the air, walls, and equipment inside the theater and increasing risk of transmission. To reduce this risk, the smoke evacuation and filtration systems are to be employed.

Types of filters:

There are mainly the high-efficiency particulate air filters that have a minimum $99.97 \%$ efficiency for filtering particles $\geq 0.3 \mu$ diameter. The ultra-low particulate air (ULPA) filters can remove $99.999 \%$ of particles minimum size of $0.05 \mu$. The size of the COVID-19 has been reported typically between 0.7 and $0.9 \mu$. The ULPA filter is ideally recommended given its minimum size of particle filtration as the viral fragments may get filtered as well. Small particles $<2 \mu$ can reach up to the respiratory bronchioles and alveoli whereas larger particles get deposited in the nose and trachea. ${ }^{17,18}$

Passive filters:

1. They essentially act like a sieve.

2. Commercially available passive filters include Laparoshield and Airofilter. ${ }^{9}$

3. A simple method is by connecting a suction tube to the cannula designated for venting out the smoke. This method is relatively simple and cost effective for smoke evacuation, especially in OT with a central suction so that aerosol dispersion does not occur into the OT sterile environment.

4. In hospitals without a central suction, bottle suction can be used but the suction machine should be placed close to the exhaust filter of the OT to enhance evacuation and a fluid trap should be set up in the suction bottle for filtration of smoke. ${ }^{9}$

5. The bottle suction can be converted into an efficient filtration system by adding a heat and moisture (HME) bacterial and viral anesthesia filter with a endotracheal tube connector and an extension tubing that can be connected to the designated port for filtration and evacuation. ${ }^{19}$ Alternatively, the HME filter can be connected to the outlet of the bottle suction, so that the air coming out of it is filtered with less viral load.

This method may theoretically filter COVID-19 viral particles. Most of these HME filters are certified for 99.99\% protection against hepatitis $B$ and hepatitis $C$ viruses that have a diameter of $0.4 \mu$ and 0.3 to $0.6 \mu$, respectively. ${ }^{20}$ Severe acute respiratory syndrome coronavirus 2 (SARS-CoV-2) has a larger diameter of 0.7 to $0.9 \mu,{ }^{21}$ hence similar filtering efficiency can be considered for the novel COVID-19 virus.

Active filters:

They have a continuous smoke evacuation and filtration mode with ULPA filters that are available commercially.

\section{Laparoscopic and Robotic Surgery}

Intraoperative recommendations:

- Keep a low intraoperative pressure (10-12 mm) when feasible.

- Port position should be according to surgeon's preference to minimize the duration of surgery and avoid any complications.

- Prevent the leakage of pneumoperitoneum during surgery by:

- Closing the cannula valves before insertion

- Minimizing the insertion, changing, and removal of instruments

- Selective use of energy sources at a low-power setting and for lesser desiccation time to reduce surgical plume

- It is essential to have a closed-circuit suction evacuation and filtration system with ULPA filter. ${ }^{22,23}$

- When performing specimen extraction through the vagina, preferably deflate the abdomen before retrieving the specimen so as to minimize the sudden spray of aerosols and the vaginal assistant should wear proper PPE including protection for the entire face. ${ }^{1,24}$

\section{Hysteroscopic Procedures}

Preprocedural screening should be done as for laparoscopy. The usage of office hysteroscopy which is an outpatient procedure should be used whenever applicable as duration of hospital stay can be reduced. Conscious sedation and regional anesthesia can be opted for when planning for operative hysteroscopy.

The risk of surgical smoke and aerosol generation is theoretically low during hysteroscopic procedures as the volatile gases dissolve in the distension medium. An active suction should be connected to the outflow especially while performing operative procedures with smoke generation (R). 
Appropriate selection of instrument is important for efficient and faster procedure. It would be wise to prefer non-smoke-generating devices such as hysteroscopic scissors and graspers.

Postoperative follow-up can be done via teleconsultation.

\section{Conclusion}

To conclude, there is no evidence as of date implicating increased spread of COVID-19 virus via laparoscopy in comparison with traditional laparotomy or vaginal surgeries. However, universal precautions and above suggestions, if meticulously followed, can have a significant role in ensuring the safety of health-care personnel. Ultimately, the care should be individualized to the patient depending on associated risk factors and available resources to have a healthy outcome for the patient as well as the treating doctor.

\section{Conflict of Interest}

None.

\section{References:}

1 AAGL-Elevating Gynecologic Surgery. Joint Society Statement on Elective Surgery during COVID-19 Pandemic. Available at: https://www.aagl.org/news/covid-19-joint-statement-onelective-surgeries/. Published March 2020. Accessed March 25, 2020

2 Ai T, Yang Z, Hou H, et al. Correlation of chest CT and RT-PCR testing for coronavirus disease 2019 (COVID-19) in China: a report of 1014 cases. Radiology 2020;296(2):E32-E40

3 Bernheim A, Mei X, Huang M, et al. Chest CT findings in coronavirus disease-19 (COVID-19): relationship to duration of infection. Radiology 2020;295(3):200463; [Epub ahead of print]

4 Li Y, Yao L, Li J, et al. Stability issues of RT-PCR testing of SARS-CoV-2 for hospitalized patients clinically diagnosed with COVID-19. J Med Virol 2020;92(7):903-908

5 Liu R, Han H, Liu F, et al. Positive rate of RT-PCR detection of SARS-CoV-2 infection in 4880 cases from one hospital in Wuhan, China, from Jan to Feb 2020. Clin Chim Acta 2020;505:172-175

6 Xiao AT, Tong YX, Zhang S. False negative of RT-PCR and prolonged nucleic acid conversion in COVID-19: rather than recurrence. J Med Virol 2020; [Epub ahead of print]

7 Gupta N, Agrawal H. COVID 19 and laparoscopic surgeons, the Indian scenario-Perspective. Int J Surg 2020

8 Xu B, Xing Y, Peng J, et al. Chest CT for detecting COVID-19: a systematic review and meta-analysis of diagnostic accuracy. Eur Radiol 2020;79:165-167
9 IAGE Good Clinical Practice Recommendations. (Version 1:16th April 2020) https://iageguidelines.blogspot.com/. Accessed August 19, 2020

10 Joint RCOG/BSGE Statement on gynaecological laparoscopic procedures and COVID-19. Available at: https://www.bsge. org.uk/news/joint-rcog-bsge-statement-ongynaecologicallaparoscopic-procedures-and-covid-19

11 AMASI. (Association of Minimal Access Surgeons of India) Guidelines for Conducting Minimal Access Surgery during COVID-19 Pandemic, (April 2020) released in https:// www. amasi.org/. Accessed Aug 19, 2020

12 Taffinder NJ, Cruaud P, Catheline JM, Bron M, Champault G. Bacterial contamination of pneumoperitoneum gas in peritonitis and controls: a prospective laparoscopic study. Acta Chir Belg 1997;97(5):215-216

13 Kwak HD, Kim SH, Seo YS, Song KJ. Detecting hepatitis B virus in surgical smoke emitted during laparoscopic surgery. Occup Environ Med 2016;73(12):857-863

14 Zhou Q, Hu X, Zhou J, Zhao M, Zhu X, Zhu X. Human papillomavirus DNA in surgical smoke during cervical loop electrosurgical excision procedures and its impact on the surgeon. Cancer Manag Res 2019;11(11):3643-3654

15 Fan C, Lei D, Fang C, et al. Perinatal transmission of COVID-19 associated SARS-CoV-2: should we worry? Clin Infect Dis 2020; $\cdots$ :ciaa22610.1093/cid/ciaa226

16 Chen Y, Chen L, Deng $\mathrm{Q}$ et al. The presence of SARSCoV-2 RNA in the feces of COVID-19 patients. J Med Virol 2020;92(7):833-840; [Epub ahead of print] 10.1002/jmv.25825

17 Taravella MJ, Viega J, Luiszer F, et al. Respirable particles in the excimer laser plume. J Cataract Refract Surg 2001;27(4):604-607

18 Ulmer BC. The hazards of surgical smoke. AORN J 2008;87(4):721-734, quiz 735-738

19 Mintz Y, Arezzo A, Boni L, Chand M, Brodie R, Fingerhut A; the Technology Committee of the European Association for Endoscopic Surgery. A low-cost, safe, and effective method for smoke evacuation in laparoscopic surgery for suspected coronavirus patients. Ann Surg 2020;272(1):e7-e8

20 Medical Advisory Secretariat. Air cleaning technologies: an evidence-based analysis. Ont Health Technol Assess Ser 2005;5(17):1-52

21 SO 29463-1:2017 High Efficiency Filters And Filter Media For Removing Particles From Air-Part 1: Classification, Performance, Testing, And Marking. https://www.iso.org/obp/ui/\#iso:std:iso:29463:-1:ed-2:v1:en.3. Accessed Aug 19, 2020

22 Englehardt RK, Nowak BM, Seger MV, Duperier FD. Contamination resulting from aerosolized fluid during laparoscopic surgery. JSLS 2014;18(3):e2014.00361

23 Angioni S. Laparoscopy in the coronavirus disease 2019 (COVID-19) era. Gynecol Surg 2020. Available at: https://doi. org/10.1186/s10397-020-01070-7. Accessed August 1, 2020

24 https://esge.org/wp-content/uploads/2020/04/ESGEGynaecological-surgery-during-Covid-outbreak-Final-27April-2020.pdf. Accessed August 1, 2020 